\title{
Isolated fallopian tubal torsion with underdiagnosed entity: a case report
}

\author{
Seon Hee Park, Jisun Yun, Jae Eun Chung*
}

Department of Obstetrics and Gynecology, National Health Insurance Service Ilsan Hospital, Goyang, Republic of Korea

Received: 05 January 2022

Accepted: 21 January 2022

\author{
*Correspondence: \\ Jae Eun Chung, \\ E-mail: obgy0278@nhimc.or.kr
}

Copyright: ( $)$ the author(s), publisher and licensee Medip Academy. This is an open-access article distributed under the terms of the Creative Commons Attribution Non-Commercial License, which permits unrestricted non-commercial use, distribution, and reproduction in any medium, provided the original work is properly cited.

\begin{abstract}
Isolated fallopian tubal torsion refers to twisting only the tube without involving the ipsilateral ovary. This is a rare type of adenexal torsion and is a gynecological emergency. Since this disease mostly occurs in women of childbearing age, accurate diagnosis and appropriate treatment are more important issues. It is known that ovarian cyst torsion occurs well in sizes greater than $5 \mathrm{~cm}$, but the epidemiology of isolated fallopian tubal torsion is unknown. Even clinically, in patients complaining of abdominal discomfort, a small andexal cyst is difficult to consider as a cause of pain. We presented an isolated fallopian tubal torsion case that can be twisted despite its small size.
\end{abstract}

Keywords: Tubal torsion, Fallopian torsion, Adnexal torsion, Acute abdomen

\section{INTRODUCTION}

An adnexal torsion is one of the gynecological emergency diseases that cause acute abdomen. Since this disease is related to the preservation of fertility, it is a gynecologic emergency. This is most often due to an ovarian mass, but very rarely is due to an only fallopian tube with a probability of one in $1.5000000 .{ }^{1}$ The risk factors for ovarian cyst torsion are relatively well known. Risk factors for ovarian torsion include ovarian enlargement. It is more common greater than $5.0 \mathrm{~cm}$ diameter. ${ }^{2}$ On the other hand, the understanding entity of fallopian tubal torsion is lack in clinical.

Here, we were reporting an isolated fallopian tubal torsion that may be clinically underdiagnosed or missed due to its small size and cystic features.

\section{CASE REPORT}

A 47-year-old woman (multiparous, gravida 3, para 2) presented to emergency department with acute right lower abdominal pain that had started suddenly 12 hours ago.
She had intrauterine device and denied any possibility of being pregnant. She had no another symptom without abdominal pain. The patient was diagnosed with a right ovarian cyst about $4 \mathrm{~cm}$ in size on a gynecological ultrasound examination performed about a month ago. The patient had surgical history including caesarean section and Roux-en-Y choledochojejunostomy and had no medical history.

On physical examination, the abdomen was flat and soft, but direct tenderness was right lower quadrant and suprapubic area without guarding and rebound tenderness. A urine pregnant test was negative and the blood test showed no specific findings. An abdomen and pelvic computed tomograph (APCT) were taken to differentiate acute abdomen. The APCT showed about $4.0 \mathrm{~cm}$ sized cystic lesion was suspected in the right adnexa with adjacent tubular structure. And pelvic peritoneal fat stranding, mainly at the right side was noted (Figure 1).

Diagnostic laparoscopy was performed under suspicion of right adnexal torsion. The surgical findings, the right tube, measuring about $4.0 \mathrm{~cm}$ by $3.0 \mathrm{~cm}$ was twisted three times 
and completely black. So, it had already progressed necrosis and ischemia (Figure 2). However right ovary was not involved and grossly normal finding. After detorsion of right fallopian tube, there was no improvement color. The patient underwent right salpingectomy (Figure 2D).

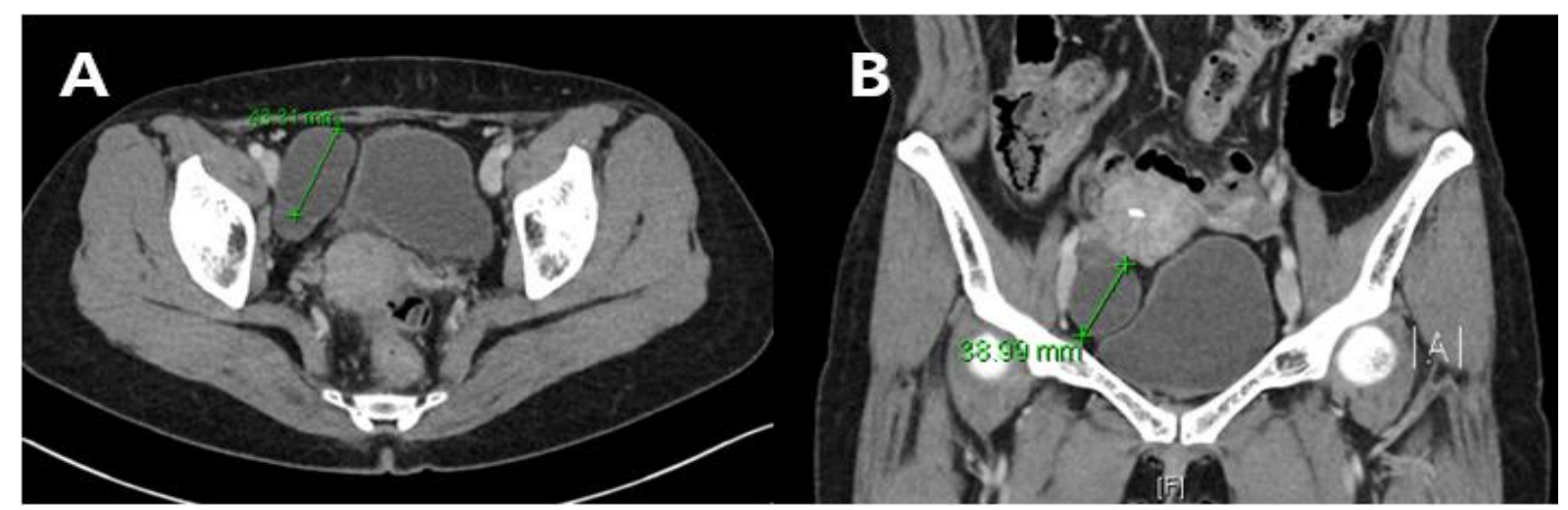

Figure 1: Two CT of pelvis showing a $4.0 \times 4.0 \mathrm{~cm}$ sized cystic structure arising from right adnexa; (A) axial plane CT pelvis image; (B) coronal plane CT pelvis image.

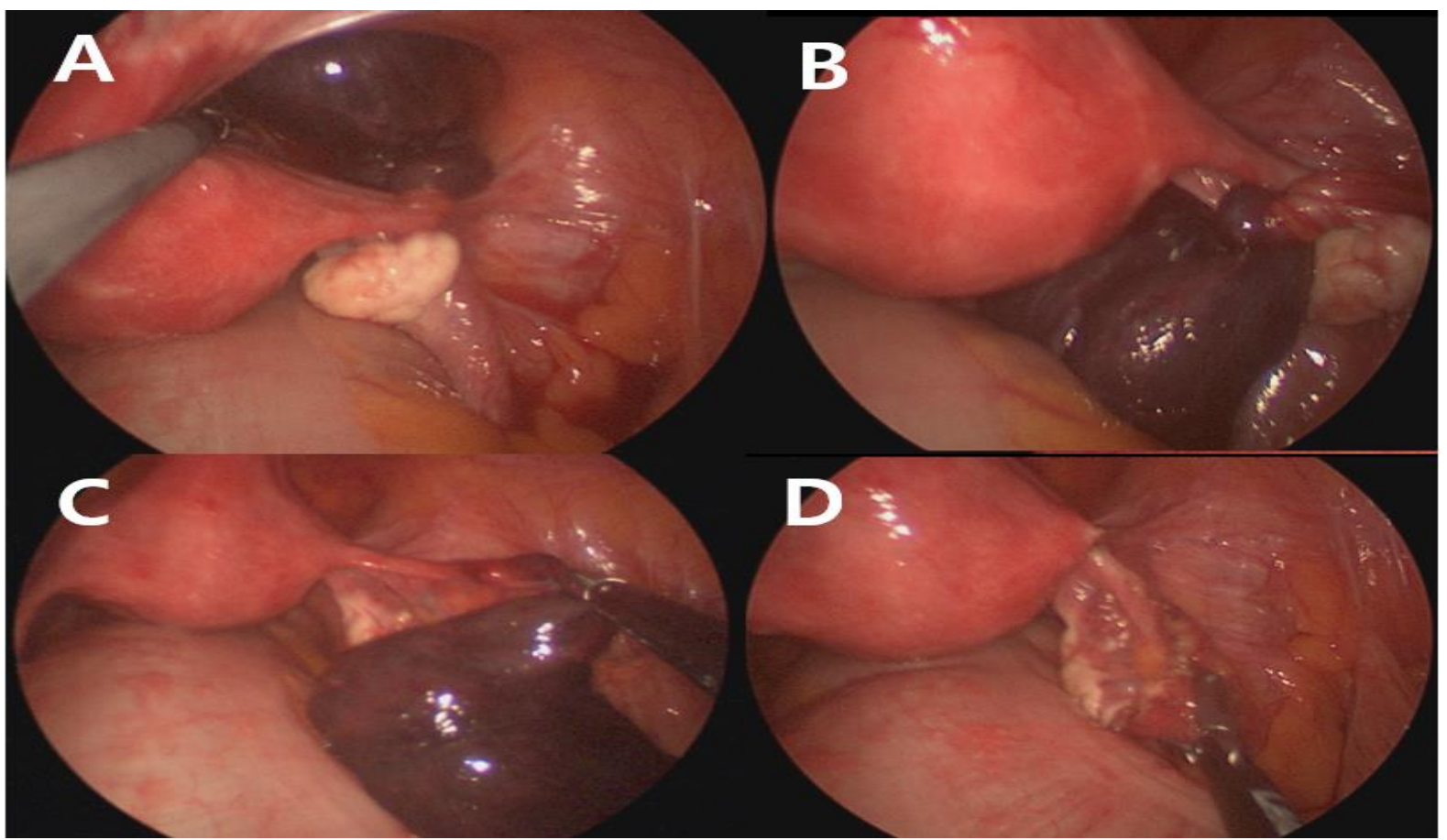

Figure 2: Four laparoscopic pictures of right fallopian tubal torsion.

The pathologic diagnosis was consistent with hydrosalpinx and torsion. The patient recovered well after surgery and was discharged without any specific findings.

\section{DISCUSSION}

Isolated fallopian tubal torsion without involvement of ovaries is an uncommon emergency, and its epidemiology is still unclear. It is more common in women of reproductive age. 3 Therefore, in this emergency disease, fertility preservation was also included as an important issue. Surgery varied depending on the time of diagnosis. If treatment was performed without delay, detorsion and paratubal cystectomy can be performed, but salpingectomy cannot be avoided if there was already irreversible damage.

The etiology of fallopian tubal torsion had not been well studied. One study reported on intrinsic and extrinsic factors for this. Intrinsic factors included congenital anomaly (excessive length of tube or spiral course), acquired pathology (hydrosalpinx, hematosalpinx, 
neoplasm and surgery), and autonomic dysfunction and abnormal peristalsis. Extrinsic factors included changes in the neighboring organs (neoplasm, adhesions and pregnancy), mechanical factors, movement or trauma to the pelvic organs and pelvic congestion. ${ }^{4}$ In our case, there was an intrinsic factor accompanied by hydrosalpinx.

The most common and typical symptom was abdominal pain and radiating pain in the ipsilateral thigh and flank was sometimes accompanied. Other symptoms may include nausea, vomiting or urinary urgency..$^{5}$ Most patients have normal vital signs and mild fever may be present. On physical examination, there was tenderness at the lesion site and not peritoneal sign. Cervical motion tenderness may be seen on bimanual pelvic examination. The patient had tenderness in the right lower quadrant (RLQ) or left lower quadrant (LLQ) area depending on the site of onset. Therefore, it was necessary to differentially diagnose acute appendicitis for RLQ pain and sigmoid disease for LLQ pain.

Laboratory values did not have diagnostic criteria, but may indicate leukocytosis or elevated C-reactive protein when the disease progresses and necrosis. However, there was no correlation between necrosis and leukocytosis. Ultimately, laboratory findings were not diagnostic tools. ${ }^{4}$

Imaging diagnostics included gynecological ultrasound sonograph, APCT and magnetic resonance imaging (MRI). Sonographic findings showed stromal edema and heterogenous appearance. Although color Doppler tracing was said to be diagnostically helpful, there were cases where it had a normal Doppler finding. ${ }^{6,7}$ Whirlpool sign and break sign were a typical sign for torsion diagnosis, but it was difficult to confirm on an ultrasound examination. On APCT, asymmetric adnexal enlargement was the most common finding. An adnexal enhancement on the torsion site was decreased. And others were a twisted vascular pedicle, pelvic fluid, inflammatory change adjacent to lesion. Although MRI was not considered as a first-line imaging study, it can be helpful in cases where it was difficult to identify. ${ }^{2,6}$ Evaluation of multi-sectional T2WI images may increase the diagnosis probability. $^{8}$

Even with these diagnostic tools, there was no typical diagnostic tool, so the diagnosis was delayed. According to previous studies, in the case of tubal torsion, the diagnosis was often delayed for more than 60 hours. ${ }^{1,2}$

An interesting and noteworthy thing about our case was that fallopian tube was twisted despite its small size. If a patient with abdominal pain showed a small cyst of only 4 $\mathrm{cm}$ or less as a result of an ultrasound sonograph or CT scan, the possibility of torsion can be underestimated or missed. In particular, abdominal tenderness was right iliac fossa area like our case, it may be more difficult to differentiate from appendicitis.

\section{CONCLUSION}

Isolated fallopian tubal torsion is uncommon and the diagnosis may be delayed due to a lack of understanding of its disease entity. Delayed diagnosis can lead to irreversible damage even with detorsion. We present a case even small-sized cysts can be torsion and cause abdominal pain. In patients with abdominal pain, a small cyst should not be ignored. Because it could be an isolated fallopian tubal torsion causing the pain. In this case, it should be accurately diagnosed and treated through diagnostic laparoscopy.

\section{Funding: No funding sources Conflict of interest: None declared Ethical approval: Not required}

\section{REFERENCES}

1. Hagege R, Sharvit M, Hamou B, Barzilay E, Pansky M, Barel O. Isolated fallopian tube torsion: an underdiagnosed entity with debatable management. J Minim Invasive Gynecol. 2022;29(10:158-63.

2. Qian L, Wang X, Li D, Li S, Ding J. Isolated fallopian tube torsion with paraovarian cysts: a case report and literature review. BMC Women Health. 2021;21(1):345.

3. Elçi E, Sayan S, Elçi G, Koç G. Isolated fallopian tubal torsion: Reproductive age case series. J Obstet Gynaecol Res. 2021;47(7):2515-20.

4. Krissi H, Shalev J, Bar-Hava I, Langer R, Herman A, Kaplan B. Fallopian tube torsion: laparoscopic evaluation and treatment of a rare gynecological entity. J Am Board Fam Pract. 2001;14(4):274-7.

5. Ziogas AC, Thanasas IK, Oikonomou IT, Tsiamanta C. Torsion of the left fallopian tube without ovarian involvement in a 47-year-old woman: a case report. Case Rep Women Health. 2020;26:00179.

6. Lourenco AP, Swenson D, Tubbs RJ, Lazarus E. Ovarian and tubal torsion: imaging findings on US, CT, and MRI. Emerg Radiol. 2014;21(2):179-87.

7. Bora SA, Kanapathippillai R, Backos M. Isolated fallopian tube torsion: an unusual cause of acute abdominal pain. BMJ Case Rep. 2011;2011:0520114183.

8. Sakuragi M, Kido A, Himoto Y, Onishi Y, Togashi K. MRI findings of isolated tubal torsions: case series of 12 patients: MRI findings suggesting isolated tubal torsions, correlating with surgical findings. Clin Imaging. 2017;41:28-32.

Cite this article as: Park SH, Yun J, Chung JE. Isolated fallopian tubal torsion with underdiagnosed entity: a case report. Int J Reprod Contracept Obstet Gynecol 2022;11:604-6. 